\title{
Identification and Treatment of Hypovitaminosis-D among In-Patients Setting of Cavan General Hospital, Ireland and Its Implication on Different Health Perspectives and Quality of Life
}

\author{
Ishaq-Khan MR*, Muthalagu P, Yousaf A, Bashir F and Hannan K \\ Cavan General Hospital, Cavan \& Co. Cavan, Ireland
}

\begin{abstract}
Aim: : People who are at risk of developing vitamin D deficiency are not usually tested for vitamin $D$ when present to hospital. The aim of this study was to explore the incidence of Hypovitaminosis-D and its treatment in admitted patient category of Cavan General Hospital, Ireland.

Methods: This is a cross sectional study which was conducted randomly among admitted patients in medicine department at CGH from August 2016 to August 2017 . A random data was collected from total of 100 patients. All blood samples were stored and labeled properly in the CGH Hematology lab till further blood profile analyzed. Data was statistically analyzed through using DSAASTAT (Onofri, Italy).

Results: Selected population of 100 patients expressed almost equal numbers in gender distribution including males and females at $51 \%$ and $49 \%$ respectively. The data was analyzed on the basis of age and gender distribution. Selected Population was divided in 3 age groups. There were 9 patients under the age-group of 30-50 years, 47 of 51-80 years and 44 of $>80$ years age-group. Among these patients, males comprised of $57 \%$ with hypovitaminosis D (vitamin D deficiency) and females were $43 \%$ respectively. Out of these 100 patients, 33 people were recorded with normal vitamin D level and 67 patients found to have low vitamin D level. These 67 patients with low vitamin D levels were selected for treatment with vitamin $D$ as per guidelines. Out of these 67 patients, unfortunately 3 people died, 11 lost their follow up, so remaining 53 patients participated in receiving treatments. Out of those 53 patients with low vitamin D, there were 29 males and 24 females. After receiving treatment with vitamin $D, 40$ people \pm 0.0001 recovered, showing vitamin $\mathrm{D}$ level $>30 \mathrm{nmol} / \mathrm{L}, 9$ patients \pm 0.0002 found mildly deficient with vitamin $\mathrm{D}$ levels between $21-30 \mathrm{nmol} / \mathrm{L}$ and 4 patients \pm 0.0003 were severely deficient with levels of $<10 \mathrm{nmol} / \mathrm{L}$. All those people with severe deficiency were more than 80 years-of-age and also were not sure about their compliance with medications. The probability value was $p \leq 0.05$
\end{abstract}

Conclusion: This study was conducted to determine hypovitaminosis D (Vitamin D deficiency) in population, admitted to hospital with previous comorbid medical conditions which are prone to develop its deficiency. This study showed that $67 \%$ population has hypovitaminosis $\mathrm{D}$ with almost equal percentage in both genders. After receiving treatments with Vitamin D replacement, almost majority (>90\%) showed recovery of vitamin D levels with very small number $(8 \%)$ showed persistent severely low levels..

Keywords: Cavan General Hospital (CGH) • Vitamin D deficiency (VDD) • Traffic Independent Logical Topology-Design Algorithm (TILDA) • Hypovitaminosis D (HVD) • DSAASTAT

\section{Introduction}

Vitamin $D$ is important for the development of healthy bone, muscles and teeth and low levels are linked to illnesses including rickets in children and osteoporosis in adults. Evidence is also emerging that vitamin D may help reduce the risk of bowel cancer and other cancers and may be linked to a number of other diseases. With this in mind it is important to make sure, vitamin $D$ level is sufficient because sun exposure and eating a healthy diet can get much of the vitamin D. But over exposure to UV radiation can also increase risk of skin cancer. There are a number of people who are at risk of having low vitamin D. They include those who have dark skin color, those who cover up their bodies when going outside, elderly people who don't go out much and have less sun exposure, women who are pregnant and who have certain disorders in the metabolism of vitamin $D$.

The aim of research was to diagnose vitamin D deficiency in people who are at risk of developing its deficiency and its timely treatment in order

\footnotetext{
*Address for Correspondence: Khan MR, Doctor, Cavan General Hospital, Cavan. Co. Cavan, Ireland, Tel: +353894167301; E-mail: dr.rizwan01@gmail.com
}

Copyright: (c) 2020 Khan MR, et al. This is an open-access article distributed under the terms of the creative commons attribution license which permits unrestricted use, distribution and reproduction in any medium, provided the original author and source are credited. to reduce the risk of complications associated with hypovitaminosis $D$. It has been established the fact that patients who present to hospital with multiple comorbidities and quite younger populations, are not tested regularly in order to diagnose hypovitaminosis $\mathrm{D}$ as holistic approach.

It has been suggested to hospital administration and other hospital medical groups that policies should be developed in order to address hypovitaminosis $\mathrm{D}$ and timely intervention can prevent multiple complications.

Vitamin D insufficiency affects almost $50 \%$ of the population worldwide. An estimated almost 1 billion people worldwide, across all ethnicities and age groups, have Vitamin D Deficiency (VDD). This pandemic of hypovitaminosis D can mainly be attributed to lifestyle (for example, reduced outdoor activities) and environmental (for example, air pollution) factors that reduce exposure to sunlight, which is required for Ultra Violet-B (UVB)-induced vitamin D production in the skin. High prevalence of vitamin $D$ deficiency is particularly an important public health issue because hypovitaminosis $D$ is an independent risk factor for increasing mortality in the general population [1].

Vitamin D has attracted much scientific interest in recent years, mostly due to its newly described roles in metabolism regulation and cell proliferation. Hypovitaminosis $D$ is associated with inadequate nutritional intake of vitamin $D$ [2]. Inadequate sunlight exposure is also a potential cause of hypovitaminosis $D$ especially in northern parts of Ireland [3]. Along with hypovitaminosis D, the incidence of obesity has risen and has become a public health concern [4]. Certain liver, kidney and hereditary disorders are associated with limiting vitamin D absorption [5].

Abnormal metabolism of vitamin $\mathrm{D}$ affects parathyroid glands and diseases 
of these glands are responsible for abnormal vitamin D metabolism as well. In primary hypoparathyroidism, there are low serum 1, $25(\mathrm{OH}) 2 \mathrm{D}$ levels, and lower intestinal calcium absorption which aggravates hypocalcaemia that leads to hypovitaminosis $\mathrm{D}$ in both children and different age groups of adults [6].

In dark skinned individuals, melanin reduces the skin's ability to make vitamin $D$ in response to sunlight exposure. Studies have showed in the past that elderly adults with dark skin are at high risk of developing vitamin $D$ deficiency [7]. Vitamin $D$ deficiency impairs bone mineralization, leading to bone softening diseases as rickets in children, osteomalacia and osteoporosis in adults [8].

Medications that accelerate metabolism of vitamin D such as Phenytoin can interfere with vitamin $D$ and bone metabolism. Drugs that activate the pregnane $\mathrm{X}$ receptor can disrupt vitamin $\mathrm{D}$ metabolism and vitamin $\mathrm{D}$ function [9]. In case of vitamin D dysfunction, muscle aches, rickets, periodontitis and weakness in limb girdles can cause difficulty in walking [10,11]. Moreover, It is found to be associated with the advancement of cancers: e.g. Cancers of breast, colon, ovarian, and prostate [12]. In vitamin D deficiency, there is reduced intestinal absorption of calcium and phosphorus that may results in hypophosphatemia and hypocalcaemia. Hypocalcaemia causes secondary hyperparathyroidism as in chronic kidney disease, which leads to phosphaturia, demineralization of bones, and when prolonged, osteomalacia and rickets [13].

The clinical manifestation of vitamin $D$ deficiency depends upon severity and duration of deficiency. Local CGH vitamin D lab values and its interpretation marked as: Normal value between 30-100 nmol/L, Mild deficiency: 20-30 $\mathrm{nmol} / \mathrm{L}$, Moderate deficiency: $11-20 \mathrm{nmol} / \mathrm{L}$, Severe deficiency $<10 \mathrm{nmol} / \mathrm{L}$.

\section{Materials and Methods}

\section{Selection of hypovitaminosis D patients}

The purpose of this study was the determination of hypovitaminosis $D$ in patients who were admitted in CGH during August 2016 to August 2017 from county Cavan, Ireland. The authors selected the random people who presented to the hospital with multiple comorbidities having tendency to develop hypovitaminosis $\mathrm{D}$. This study has been carried out on the basis of hypovitaminosis $D$ prevalence, association with gender, different age groups and underlying different co-morbid conditions. As per results, treatment for hypovitaminosis $D$ was also suggested according to guidelines and protocols.

\section{Sample collection}

This cross sectional study was conducted randomly in patients admitted in medicine department at CGH from August 2016 to August 2017. Total 100 patients of both genders were included in this study. A random data was collected from total of 100 patients.

\section{Sample storage}

Blood samples were drawn and stored at $-80^{\circ} \mathrm{C}$ in local CGH lab till further routine investigations. The authors obtained consent from all those people after explaining the purpose and implications of study.

\section{Data analysis}

Data was statistically analyzed using DSAASTAT (Onofri, Italy). This study was conducted at CGH over the given period of time.

\section{Results}

According to age distribution, 9 cases of hypovitaminosis $D$ were found among 30-50 years age group, 47 people recorded in 51-80 year's group along with 44 reported above 80 years of age. However, according to gender distribution, the total number of females comprised with hypovitaminosis $D$ was $49 \%$ and males were $51 \%$. According to percentage value, $12 \%$ were reported with severe hypovitaminosis D, $52 \%$ moderate and $36 \%$ reported as mildly deficient. The significance of vitamin $\mathrm{D}$ according to gender distribution data describe that $33 \%$ patients were normal with vitamin D levels and $67 \%$ were reported with low levels.

The classification of hypovitaminosis $D$ was described according to local CGH lab values (normal value of $30-100 \mathrm{nmol} / \mathrm{L}$ ): mild deficiency (insufficiency) with $21-29 \mathrm{nmol} / \mathrm{L}$, moderate deficiency at $11-20 \mathrm{nmol} / \mathrm{L}$ and severe deficiency with levels $<10 \mathrm{nmol} / \mathrm{L}$.

The study showed equal gender population selected in this study including females and males $49 \%$ and $51 \%$ respectively. Only $33 \%$ were found to have normal levels whereas, $67 \%$ patients were found to have Hypovitaminosis D. Therefore, males (57\%) were predominantly deficient while females were $43 \%$. However, $12 \%$ found to be severely deficient, while $52 \%$ versus $36 \%$ were characterized as moderate and mildly deficient respectively. Further, analysis was done after 6 month's treatment with Vitamin D3 (Cholecalciferol) according to dose required as per levels (Figure 1 ).

The treatment for severe hypovitaminosis $D$; replacement started orally with vitamin D3 at 50,000 IU once weekly for 6 to 8 weeks, followed by $800 \mathrm{IU}$ daily (Thorens drops, Altavita D3, Desunin). Moderate deficiency; replacement started with 800 to 1000 IU daily (Stirivit D3, Desunin, Fultium D3) and 600 to $800 \mathrm{IU}$ once daily was given in mild deficiency which may be sufficient to maintain levels in the target range. For patients with malabsorption; high doses of vitamin $D, 10,000$ to 50,000 units daily may be necessary to replete in gastrostomy or malabsorption.

Artificial UV B radiation exposure from tanning beds (Sunbeds, sunlamps) is effective in increasing and maintaining serum $25(\mathrm{OH}) \mathrm{D}$ levels. However, because there are no defined safe exposure limits for UVB exposure, so it is not typically used to treat vitamin $D$ deficiency. One possible exception is patients with malabsorption who remain vitamin $D$ deficient even with high dose oral supplementation (50,000 international units daily). In such patients, serum $25(\mathrm{OH}) \mathrm{D}$ levels should be monitored to determine the optimal UVB dose and frequency.

\section{Distribution of total patients after treatment}

Total number of patients was 67 and out of this, 3 died and 11 lost their follow up. So, 53 patients participated in receiving treatments comprising 29
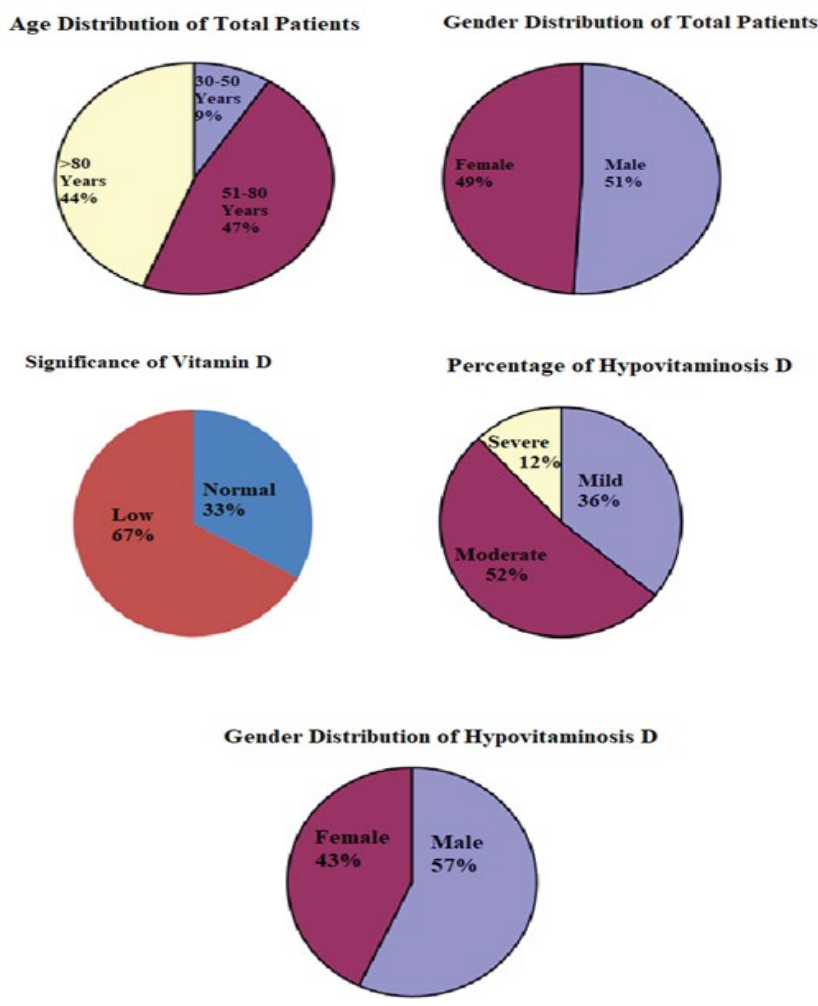

Figure 1. Significance of Vitamin D before treatment. 


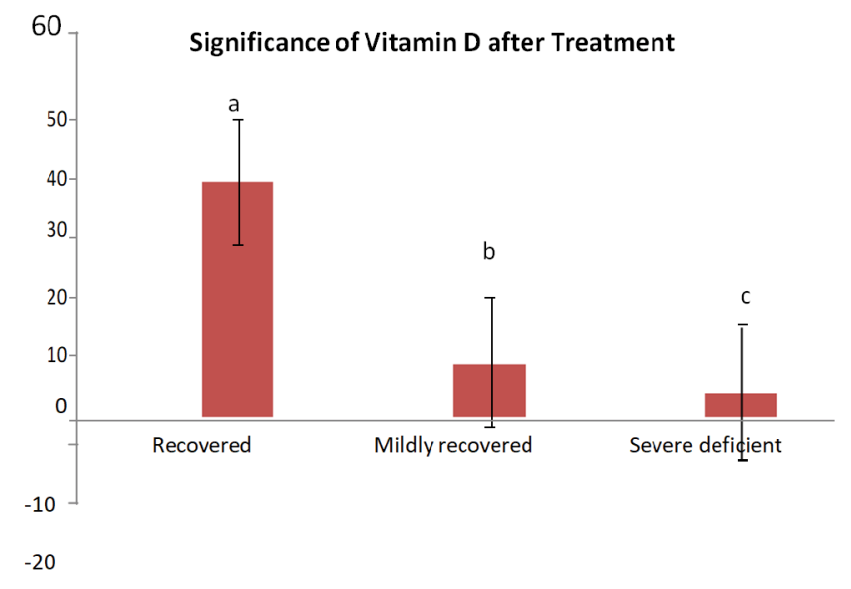

Figure 2. Graph showing significance of Vitamin D after treatment.
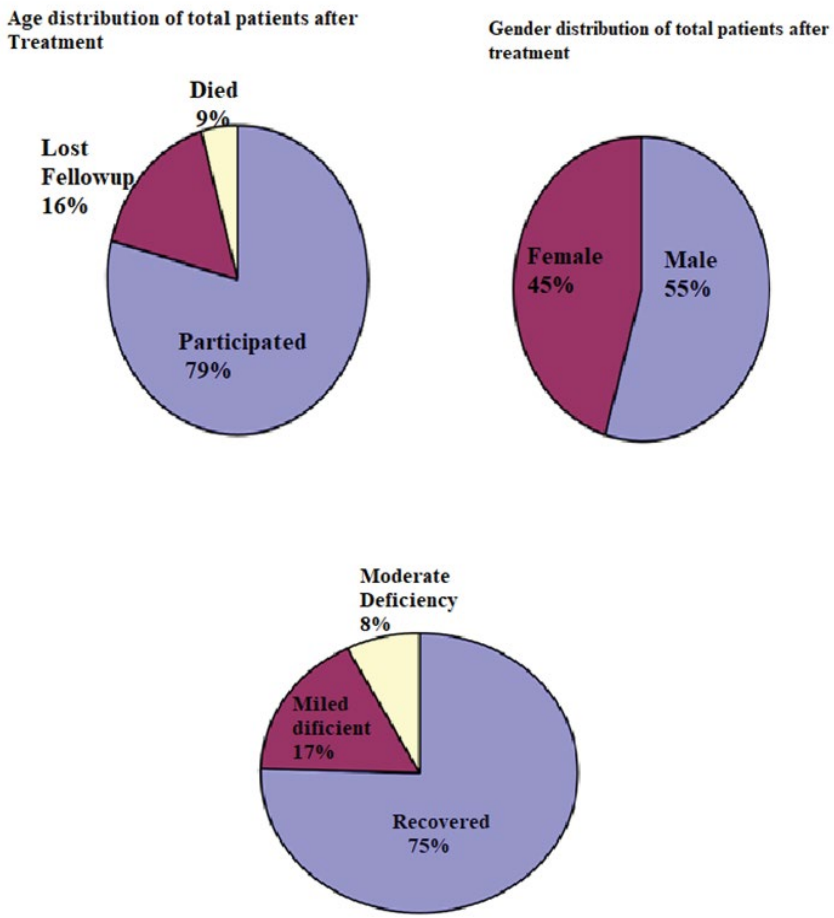

Figure 3. Significance of Vitamin D after treatment.

Table 1. Effect of rainfall on Vitamin D.

\begin{tabular}{cccc}
\hline Age & Male & Female & Pregnancy+ Lactation \\
\hline $0-12$ Months & $400 \mathrm{IU}$ & $400 \mathrm{IU}$ & - \\
\hline $1-70$ Years & $600 \mathrm{IU}$ & $600 \mathrm{IU}$ & $600 \mathrm{IU}$ \\
\hline$>70$ Years & $800 \mathrm{IU}$ & $800 \mathrm{IU}$ & - \\
\hline
\end{tabular}

male and 24 females. Out of total 53,40 patients (75\%) recovered with vitamin D level $>30 \mathrm{nmol} / \mathrm{L}, 9$ patients $(17 \%)$ recorded mildly deficient with vitamin D level of 21-30 nmol/L and only 4 patients (8\%) showed severely deficient levels $<10 \mathrm{nmol} / \mathrm{L}$. All those with severe deficiency were noticed more than 80 years of age and there was also query about their compliance status (Figure 2). The results have expressed the fact that after treatment with vitamin $D$, recovered level of vitamin $D$ showed $40 \% \pm 0.0001$ following mild deficient vitamin-D with $9 \% \pm 0.0002$ and severely deficient was $4 \% \pm 0.0004$ (Figure 3).

\section{Discussion}

The vitamin $D$ deficiency depends upon life style, socio-economic status and different parts of Ireland. Although there is discrepancies in the results of cross-sectional studies that compare vitamin $D$ status in hypovitaminosis $D$ patients and healthy individuals, the high prevalence of vitamin $D$ deficiency in general population including hypovitaminosis $D$ patients is predominantly accepted. Thus, there was a general recommendation of vitamin D assessment in all hypovitaminosis $D$ patients especially early after diagnosis and in first demyelinating event. The normal range of $25(\mathrm{OH}) \mathrm{D}$ have been revised in recent years. However, the normal range stands between 30 and $100 \mathrm{ng} / \mathrm{ml}$ (75 and $250 \mathrm{nmol} / \mathrm{L}$ ) [14]. Less than $10 \mathrm{ng} / \mathrm{ml}$ is considered as deficiency and a range between 11 and $30 \mathrm{ng} / \mathrm{ml}$ considered as insufficiency [15]. However for many of extra bone disorders of vitamin $D$ including hypovitaminosis $D$ value of $40 \mathrm{ng} / \mathrm{ml}(100 \mathrm{nmol} / \mathrm{L})$ was described [16]. The study showed by Trinity College Dublin that there should be some foods which can be fortified to obtain recommended dietary amount of vitamin D [17]. The recommended dietary allowances for Vitamin $D$ describe as average daily intake of sufficient vitamin $D$ to meet the nutrient requirements of nearly all (97\%-98\%) healthy people [18]. There was a study conducted in Ireland to determine the level of vitamin D above 50 years of age and there were $12.3 \%$ of population involved in hypovitaminosis D [19]. Another study reported an important factor in hypovitaminosis $D$ is seasonal variation in which winter months reported more hypovitaminosis $D$ cases than summers with (21.9\% vs. $4.4 \%)$ ratio [20]. However, it was also revealed that northern areas in Ireland suffered more with hypovitaminosis $D$ cases due to more rainfall than southern areas where the weather is more sunny and shiny [21] (Table 1).

According to various studies worldwide different countries of Europe comprised different levels of hypovitaminosis $D$ in the older population according to their standard vitamin $D$ values. The level of hypovitaminosis $D$ in Ireland's older population $>50$ years of age comprised $13.1 \%$ which is $<30$ $\mathrm{nmol} / \mathrm{L}$ that is comparatively high as English $12.3 \%$ and Dutch $11 \%$ in $>65$ years of age with least levels of $25 \mathrm{nmol} / \mathrm{L}$ [22]. Surprisingly, hypovitaminosis D level was observed in elderly population of Germany at $30.2 \%$ and Iceland $17.1 \%$ respectively [23]. However, hypovitaminosis $D$ is not only dependent upon climatic and latitude but in other countries of the same latitude and climate the prevalence of hypovitaminosis $D$ is lower. In Swedish studies of older population including both men and women, the mean vitamin $D$ level was $95.0 \mathrm{nmol} / \mathrm{L}$ and $69.0 \mathrm{nmol} / \mathrm{L}$ respectively [24]. This is in comparison to $50.6 \mathrm{nmol} / \mathrm{L}$ for men and $52.5 \mathrm{nmol} / \mathrm{L}$ for women in TILDA [25]. This contrast has been observed in vitamin $\mathrm{D}$ level among Irish population and other Nordic countries because of the consumption of vitamin $D$ fortified supplements in ratio $8.3 \%$ and $50 \%[26,27]$. The current data showed that patients above age of 80 years were suffering more with hypovitaminosis $D$ following patients of 50 to 80 years of age were on second position with vitamin $D$ deficiency. The patients from 21 to 50 years of age were reported moderate to mild with vitamin $D$ deficiency. The main factor of vitamin $D$ deficiency in elderly population that they are socially inactive, live on their own, have poor diet, less consumption of fortified vitamin D supplements and poor life style. Vitamin D deficiency has been associated with numerous health outcomes, including risk of rickets in children or osteomalacia in adults, increased risk of fractures, falls, cancer, autoimmune disease, infectious disease, type 1 and type 2 diabetes, hypertension, heart diseases, and other diseases such as multiple sclerosis [28].

In case of severe Vitamin $D$ deficiency, replacement was started with weekly 50,000 IU of vitamin D3 orally for 6 to 8 weeks, and 800 IU daily thereafter (Thorens drops, Altavita D3, Desunin). Moderate deficiency, replacement started with 800 to 1000 IU daily (Stirivit D3, Desunin, Fultium D3) whereas in mild deficiency, replacement was started with 600 to $800 \mathrm{IU}$ daily that may be sufficient to maintain levels in target range. For patients with malabsorption, high doses of vitamin $D$ of 10,000 to 50,000 units daily may be necessary to replete in gastrostomy or malabsorption. Artificial UV-B radiation exposure from tanning beds (Sunbeds, sunlamps) is effective in increasing and maintaining serum $25(\mathrm{OH}) \mathrm{D}$ levels.

However, there are no defined safe exposure limits for UVB exposure, so it is not typically used to treat vitamin D deficiency. One possible exception is patients with malabsorption who remain vitamin $D$ deficient even with high dose oral supplementation (50,000 international units daily). In such patients, 
serum $25(\mathrm{OH}) \mathrm{D}$ levels should be monitored to determine the optimal UVB dose and frequency [29].

In Ireland $74 \%$ of adults and $88 \%$ of primary school children, have less than half of the recommended daily amount of vitamin D. Only $30 \%$ of Irish people aged $60^{+}$who fracture their hip will regain their independence. However once a person has fractured a hip, this places them at a higher risk of fracturing again. One hip fracture costs almost $€ 55,000$ in total including rehabilitation and approximately one hip fracture occurs every 30 seconds in the EU. There is recommendation that high-risk groups are screened to start vitamin $D$ supplement followed by remeasure levels at 3-4 months later to verify that target has been achieved [5].

On the basis of results of this study, following recommendations are suggested to above mentioned hospital: To check vitamin D level in all patients who are at risk or having comorbid conditions which can increase the risk for its deficiency, especially elderly people, patients of low mobility, decreased sunlight exposure, malnutrition, conditions affecting it's Gl absorption, established osteoporosis or at risk. They are advised to check its correct value with units (vitamin D service.com), start treatment as per guidelines. Follow up at 3 or 6 months either in clinic or with GP [30-32].

\section{Conclusions}

- This study was conducted to determine the hypovitaminosis $D$ in Ireland.

- Among hundred patients female comprised of $43 \%$ with hypovitaminosis D and males were $57 \%$ respectively.

- Total number of hypovitaminosis D patients were 67 from which 3 died unfortunately, 11 lost their follow up whereas, total 53 people participated in receiving treatments.

- 40 patients \pm 0.0001 recovered with vitamin $\mathrm{D}$ level $>30 \mathrm{nmol} / \mathrm{L}, 9$ patients \pm 0.0002 found mildly deficient with level between $21-30 \mathrm{nmol} / \mathrm{L}$ and 4 patients \pm 0.0004 showed severe deficient levels $<10 \mathrm{nmol} / \mathrm{L}$.

- A maintenance vitamin D treatment of 1500-2000 IU daily or equivalent intermittent (weekly, fortnightly or monthly) Dose. Fortnightly or monthly dose might have better compliance.

- When D3 is available, supplementation with D3 is preferred.

\section{Recommendations}

- $\quad$ Patients suffering with low vitamin D should consume 1000-4000 IU daily (average $2000 \mathrm{IU}$ ) of vitamin D supplements to reach level $30 \mathrm{nmol} / \mathrm{L}$.

- Sun-bath could also provide vitamin D equivalent to oral consumption of up to $20,000 \mathrm{IU}$ per day.

- Daily consumption of vitamin D2 and vitamin D3 seems to have almost similar effect on serum $25(\mathrm{OH}) \mathrm{D}$ levels. When D3 is available, supplementation with D3 is preferred as it avoids problems with differences in $25(\mathrm{OH}) \mathrm{D}$ assay specificity because D3 maintains serum $25(\mathrm{OH}) \mathrm{D}$ level consistent for a longer time when consumed as intermittent regimen.

- Patients are advised to consume vitamin $D$ enriched foods on daily basis.

- The measurement of $25(\mathrm{OH}) \mathrm{D}$ is recommended after three months of therapy, if vitamin $D$ supplements are being consumed on daily or weekly basis. However, according to some experts the recommended routine serum vitamin $D$ level needs to be tested at least two times a year especially in beginning of spring and autumn.

\section{Conflict of Interest}

There is no conflict of interest in this study.

\section{References}

1. Nair Rathish, Maseeh Arun. "Vitamin D: The "sunshine" vitamin". J Pharmacol Pharmacother (2012):118-126.

2. Daniel."Hypovitaminosis D: A common deficiency with pervasive consequences". JAAPA (2015): 20-26.

3. Carroll Aoife, Onwuneme Chike, McKenna Malachi. "Vitamin D status in Irish children and adolescents: Value of fortification and supplementation". Clin Pediatr (Phila) (2014): 1345-1351.

4. Reis de-Azevedo Fernanda, Caramelli Bruno. "Hypovitaminosis D and obesitycoincidence or consequence". US Endocrin (2013): 40-43.

5. https://www.merckmanuals.com/professional/nutritional-disorders/vitamindeficiency,-dependency,-and-toxicity/vitamin-d-deficiency-and-dependency

6. Scharla Stephan. "Diagnosis of disorders of vitamin D-metabolism and osteomalacia". Clin Lab (2008): 451-459.

7. Harris Susan. "Vitamin D and African Americans". J of Nutri (2006): 1126-1129.

8. Cummings Steven, Kiel Douglas, Black Dennis. "Vitamin D supplementation and increased risk of falling: A cautionary tale of vitamin supplements retold". JAMA Intern Med (2016):171-172.

9. Uwe Gröber, Klaus Kisters. "Influence of drugs on vitamin D and calcium metabolism". Dermatoendocrinol (2012): 158-166.

10. Wang Chin-Wei, McCauley Laurie. "Osteoporosis and Periodontitis". Curr Osteoporos Rep (2016): 284-291.

11. Joshi Devina, Center Jacqueline, Eisman John. "Vitamin D deficiency in adults". Aust Prescr (2010): 103-106.

12. Cherniack Paul, Levis Silvina, Troen Bruce. "Hypovitaminosis D: A widespread epidemic". Geriatrics (2008): 24-30.

13. Yorifuji Junko, Yorifuji Tohru, Tachibana Kenji. "Craniotabes in normal newborns: the earliest sign of subclinical vitamin D deficiency". J Clin Endocrinol Metab (2008): 1784-1788

14. Holick Michael, Binkley Neil, Bischoff-Ferrari Heike. "Evaluation, treatment, and prevention of vitamin $\mathrm{D}$ deficiency: an Endocrine Society clinical practice guideline". J Clin Endocrinol Metab (2011):1911-1930.

15. Hanley David, Cranney Ann, Jones Glenville. "Vitamin D in adult health and disease: A review and guideline statement from Osteoporosis Canada". Can Med Assoc J. 2010:610-618.

16. Płudowski Paweł, Karczmarewicz Elżbieta, Bayer Milan. "Practical guidelines for the supplementation of vitamin $D$ and the treatment of deficits in Central Europe-recommended vitamin $D$ intakes in the general population and groups at risk of vitamin D deficiency". Endokrynol Pol (2013):319-327.

17. Laird Eamon, O'Halloran Aisling, Carey Daniel. "The Prevalence of Vitamin D deficiency and the determinants of $25(\mathrm{OH}) \mathrm{D}$ concentration in older Irish adults: Data from The Irish Longitudinal Study on Ageing (TILDA)". J Gerontol A-Biol (2018): 519-525

18. Institute of Medicine, Food and Nutrition Board. "Dietary Reference Intakes for Calcium and Vitamin D". Ross Catharine, Taylor Christine, Yaktine Ann, Del Valle Heather Washington, DC: National Academy Press,USA (2010).

19. Cashman Kevin, Dowling Kirsten, Skrabakova Zuzana. "Vitamin D deficiency in Europe: pandemic?" Am J Clin Nutr (2016): 1033-1044.

20. Holick MF. "High prevalence of vitamin $D$ inadequacy and implications for health". Mayo Clin Proc. (2006): 353-373.

21. Chowdhury Rajiv, Kunutsor Setor, Vitezova Anna. "Vitamin D and risk of cause specific death: Systematic review and meta-analysis of observational cohort and randomised intervention studies". BMJ (2014): 348.

22. Kuchuk Natalia, Pluijm Saskia, Van-Schoor Natasja. "Relationships of serum 25-hydroxyvitamin $\mathrm{D}$ to bone mineral density and serum parathyroid hormone and markers of bone turnover in older persons". J Clin Endocrinol Metab (2009): 1244-1250.

23. Rabenberg Martina, Scheidt-Nave Christa, Busch Markus. "Vitamin D status among adults in Germany-results from the German Health Interview and Examination Survey for Adults (DEGS1)". BMC Public Health. (2015): 641. 
24. Hagstrom Emil, Hellman Per, Larsson Tobias. "Plasma parathyroid hormone and the risk of cardiovascular mortality in the community". Circulation (2009): 2765-2771.

25. Gerdhem P, Ringsberg KA, Obrant KJ, Akesson K. "Association between 25-hydroxy vitamin D levels, physical activity, muscle strength and fractures in the prospective population-based OPRA study of elderly women". Osteoporos Int (2005): 1425-1431.

26. Lamberg-Allardt Christel, Brustad Magritt, Meyer Haakon. "Vitamin DA systematic literature review for the $5^{\text {th }}$ edition of the Nordic nutrition recommendations". Food Nutr Res (2013):1-31.

27. Sigurdsson G, Franzson L, Steingrimsdottir L. "The association between parathyroid hormone, vitamin $\mathrm{D}$ and bone mineral density in 70-year-old Icelandic women". Osteoporos Int (2000): 1031-1035.

28. Basit S. "Vitamin D in health and disease". Br J Biomed Sci (2013): 161-172.

29. Jones Glenville. "Pharmacokinetics of vitamin D toxicity". Am J ClinNutr (2008):582S-586S
30. Souberbielle Jean-Claude, Body Jean-Jacques. "Vitamin D and musculoskeletal health, cardiovascular disease, autoimmunity and cancer: Recommendations for clinical practice". Autoimmun Rev (2010):709-715.

31. Henry Helen, Bouillon Roger, Norman Anthony. "14 $4^{\text {th }}$ Vitamin D workshop consensus on vitamin D nutritional guidelines". J Steroid Biochem Mol Biol. 2010:4-6.

32. Onofri Andrea. "Routine statistical analyses of field experiments by using an excel extension". In proceedings $6^{\text {th }}$ national conference Italian biometric society (2007): 93-96.

How to cite this article: Kha Muhammad Rizwan Ishaq, Muthalagu Poochellam, Anam Yousaf, Bashir Farhan and Hannan Kieran, et al. "Identification and Treatment of Hypovitaminosis-D among in-patients setting of Cavan General Hospital, Ireland and its implication on different health perspectives and quality of life." Vitam Miner 09 (2020): 189. doi: 10.37421/VTE.2020.9.189. 